\title{
Aproximaciones al análisis del discurso de las Cumbres de Estocolmo y Rio: Su influencia en la legislación colombiana y su relación con la Educación Ambiental durante las postrimerías del siglo XX
}

\section{Approaches to the Analysis of the Stockholm and Rio Summits Discourse: Its Influence on Colombian Legislation and its Relationship with Environmental Education during the Late Twentieth Century}

\author{
Diana María Perea Palomino \\ Licenciada en Ciencias Sociales. Magister en Estudios Socio ambientales. Facultad Latinoamericana en \\ Ciencias Sociales (FLACSO). Quito, Ecuador. \\ diamar210@hotmail.com | 0000-0003-0833-9095
}

Para citar este artículo: Perea Palomino, D. M. (2020). Aproximaciones al análisis del discurso de las Cumbres de Estocolmo y Rio: Su influencia en la legislación colombiana y su relación con la Educación Ambiental durante las postrimerías del siglo XX. Entorno Geográfico, (20), 127-141. https://doi.org/10.25100/eg.v0i20.10851

\section{Resumen}

El objetivo de este trabajo es explorar cómo las diferentes narrativas y regímenes de verdad estipulados en la gobernanza ambiental internacional de dos Cumbres internacionales como son Estocolmo y Rio, influyeron en el ámbito jurídico colombiano con relación a la Educación Ambiental, durante la década de los 70 y 90 del siglo XX. Para el contexto colombiano se abordará el Código Nacional de los Recursos Naturales Renovables y la Protección al Medio Ambiente de 1974, porque es a partir de esta normatividad donde empieza la construcción de espacios formativos y prospectivos para el cuidado y manejo adecuado del ambiente, así como también, la Ley 99 de 1993, la cual reúne en su totalidad, elementos contenidos en la
Declaración de Río de Janeiro de 1992 y, el Decreto 1743 de 1994, el cual reglamenta el Proyecto Escolar de Educación Ambiental (PRAE) en las instituciones educativas de Colombia.

Palabras clave: Discurso, biopolítica, biopoder, antropocentrismo, Estado.

\begin{abstract}
The objective of this paper is to explore how the different narratives and regimes of truth stipulated in the international environmental governance of two international summits such as Stockholm and Rio, influenced the Colombian legal environment in relation to Environmental Education, during the decade of the 70's and 90's of the 20th century. For the Colombian context, the National Code of Renewable Natural Resources and
\end{abstract}


Environmental Protection of 1974 will be addressed, because it is from this normative that the construction of formative and prospective spaces for the care and adequate management of the environment begins, as well as, Law 99 of 1993, which gathers in its totality elements contained in the Declaration of Rio de

Recibido: 17 de junio de 2020

\section{Introducción}

La segunda mitad del siglo XX constituye una nueva configuración del mundo que se enmarca con el periodo posbélico del año 1945, dando inicio a una nueva gobernanza a nivel global. Se perfilan organismos internacionales como la Organización de las Naciones Unidas (ONU), que ayudará a brindar posibilidades en el mantenimiento de la paz, derechos humanos, asuntos humanitarios y la conformación de una nueva gobernanza ambiental internacional. Así pues, se desarrollan distintas cumbres ambientales que insertan en su discurso a la EA como una vía para crear conciencia en los habitantes del planeta frente a los inminentes problemas ambientales globales.

En este sentido, los Estados van perdiendo su individualidad y soberanía para controlar y gestionar de manera independiente sus problemas internos, insertándose en nuevas lógicas de interrelación con otros Estados, influenciados considerablemente por el mundo exterior. A esto se le puede analizar como una nueva gobernanza de carácter internacional, donde las cuestiones ambientales no son la excepción.
Janeiro of 1992 and, Decree 1743 of 1994, which regulates the School Project of Environmental Education (PRAE) in the educational institutions of Colombia.

Keywords: Discourse, biopolitics, biopower, anthropocentrism, biocentrism, state.

Aceptado: 02 de septiembre de 2020

De acuerdo a lo anterior, se perfila un nuevo ordenamiento jurídico con una estructura jerárquica superior desde los ámbitos internacionales, constituyendo una nueva gobernanza ambiental internacional a partir de la década de los años 70 del siglo $\mathrm{XX}$, que más que establecer medidas coactivas para la protección eficiente del medio ambiente, se caracteriza por ser eminentemente preventivo, con una alta propensión de flexibilidad, buscando llegar a consensos internacionales mediante normas blandas o flexibles denominadas "soft law", en donde se establecen mecanismos de cooperación entre las partes (Cardesa Salzmann, 2011, p. 35).

En este sentido, más que establecerse normas imperativas u obligatorias, lo que se plantea son recomendaciones sobre una determinada problemática ambiental. Dichas sugerencias son discursividades que son asumidas por los Estados en sus legislaciones nacionales, tal es el caso de dos Cumbres ambientales internacionales: Estocolmo y Rio, objeto de estudio en el presente trabajo, porque han marcado hitos relevantes con relación a la preservación de la naturaleza y la cuestión del desarrollo 
sostenible, representando el inicio para la implementación de políticas, instituciones y normativas con relación al cuidado de los recursos naturales a nivel de los Estados, donde Colombia no ha sido un país ajeno en la preocupación por el medio ambiente. En este sentido, se hace pertinente plantear la siguiente pregunta de análisis ¿Cómo incidieron los discursos de las Cumbres de Estocolmo y Rio durante las décadas de los 70 y 90 del siglo XX en la legislación colombiana con respecto a la Educación Ambiental?

\section{Fundamentación teórica y conceptual}

El presente trabajo tiene un enfoque postestructuralista, rama de la teoría crítica propia de las postrimerías del siglo XX que busca superar aquellas oposiciones binarias propias del estructuralismo. De esta forma, el postestructuralismo introduce en términos de sus sistemas de signos, códigos y discursos, la dinámica entre naturaleza y sociedad como un constructo integrado y no dual marginado de la teoría social en el estructuralismo. Pensadores como Foucault, Escobar y Mendizábal aportan elementos significativos al análisis del discurso desde este enfoque:

Michel Foucault uno de los representantes del postestructuralismo, analiza dentro de sus enunciados, al "orden del discurso"; el cual siendo propio de un periodo especifico, "tiene una función normativa y regulada y pone en acción mecanismos de organización de lo real por intermedio de la producción de saberes, estrategias y prácticas" (Foucault citado en Revel, 2008, p. 50) Desde esta perspectiva, el discurso puede cumplir una función legitimadora del poder, en este caso, a través de leyes, mandatos, marcos legales, etc. Por otro lado, Arturo Escobar plantea que el discurso es la articulación del conocimiento y el poder, de lo visible y lo expresable, es el proceso por el cual la realidad social inevitablemente se hace verdad. (Escobar, 2007). Por último, Rodrigo Mendizábal analiza el discurso como "una producción social, que forma parte de un proceso a través del cual los grupos sociales intercambian y configuran sus realidades y consolidan sus concepciones de hacer realidad la vida cotidiana" (Mendizábal, 1999, p. 109).

De esta manera, el discurso como función "legitimadora del poder", como "articulación del conocimiento y el poder" y como una "producción social", incluye dentro de sus aristas, contextualizaciones espacio-temporales que involucran el lugar de enunciación, haciendo referencia al contexto social, político, cultural y económico sin desligarse de los acontecimientos históricos de la época. Así pues, los discursos abordados desde el Norte global se manejan desde otras perspectivas, otros paradigmas, otras visiones y cosmovisiones distintas a las del Sur global. Sin embargo, ciertas discursividades emergidas desde las Cumbres de Estocolmo y de Río con relación al medio ambiente y a la Educación Ambiental (de ahora en adelante EA), han sido producidas en el Norte global y reproducidas en países del Sur través de sus normatividades, sin tener en consideración que Norte y Sur son contextos asimétricos. 
Todo discurso se encuentra enfocado en diversos paquetes de lenguaje que se encuentran circulando en la sociedad; éstos se denominan textos y pueden ser literarios, no literarios, transcritos, audiovisuales y sociales. Las Cumbres analizadas en este trabajo son textos sociales porque son producto de un intercambio social, funcionando en el marco de sus coherencias que pueden ser diferentes para el enunciador y para el perceptor (Mendizábal, 1999, p. 116).

Así mismo, se hace relevante abordar otras conceptualizaciones para la deliberación discursiva de las Cumbres de Estocolmo y Rio y su influencia en el ámbito jurídico colombiano con relación a la EA. Tales conceptualizaciones tienen que ver con el Estado, el Poder, el Biopoder, la Biopolítica, la Soberanía y la Educación Ambiental.

El Estado es una categoría polisémica que está en constante evolución de acuerdo al contexto en el que se vive. En este sentido, el Estado "es una expresión posible situada dentro de un marco temporal preciso" (Foucault citado en Revel 2008, p. 60). Esta categoría polisémica ha sido considerada por algunos pensadores como una asociación o comunidad de acuerdo a Weber; como un resultado establecido por individuos por medio de un contrato social en términos de Rousseau (Aldo Isuani, 2011) y, en lenguajes marxistas, como un "órgano separado de la sociedad, un instrumento de poder y dominación de unas clases sobre otras" (Marx, citado en Calduch, 1991, p. 4). Actualmente, el Estado como una categoría de relación internacional puede ser definido como "toda comunidad humana establecida sobre un territorio y con capacidad para desarrollar sus funciones políticas $\mathrm{y}$ económicas, mediante la generación y organización de relaciones simultáneas de poder y de solidaridad que institucionaliza en su interior y proyecta hacia el exterior" (Calduch, 1991, 6).

El segundo término, el poder, es una entidad cambiante. Su esencia no puede ser analizada como algo coherente, unitario y estable, sino que de acuerdo a Foucault debe ser vista como "relaciones de poder". En efecto, “es un ordenamiento donde se cruzan las prácticas, los saberes y las instituciones, donde su objetivo no solo se reduce a la dominación, sino que no pertenece a nadie y varía en la historia. Además, cuenta con modalidades instrumentales como las armas, las disparidades económicas, los mecanismos de control, los sistemas de vigilancia y el discurso" (Foucault citado en Revel 2008, p. 114).

De acuerdo a lo anterior, el Poder a lo largo de la historia tiende a sufrir transformaciones a través del biopoder y la biopolítica. El primero hace referencia al ejercicio de los Estados modernos de "explotar numerosas y diversas técnicas para subyugar los cuerpos y controlar la población" (Foucault citado en Campos Fernández, 2010, p. 231). Para Foucault, el biopoder puede ser analizado en dos niveles: el disciplinario y el regulatorio. El primer nivel, tiene como objetivo disciplinar los cuerpos de los hombres a través de instituciones como las escuelas, las prisiones o los hospitales. El segundo nivel hace referencia a un poder 
regulatorio, el cual es ejercido por el Estado, ocupándose de la población dentro de sus límites territoriales que, a través de diversos mecanismos, busca establecer el control sobre sus ciudadanos. A este tipo de transformación de poder se le puede denominar biopolítica (Vásquez Rocca, 2013).

El término soberanía es también un concepto cambiante, que ha evolucionado con el tiempo, pues no es igual el concepto de soberanía emergido durante la época feudal, el cual se utilizó para sometimiento de unos sobre otros, o durante el siglo XVI con los estudios de Jean Bodin (15301596), quien afirmaba que la "soberanía es un poder absoluto y perpetuo, limitado únicamente por las restricciones de los gobernantes" (Citado en Becerra, Povedano y Téllez, 2016, p. 59). Así mismo, Rousseau en el siglo XVIII con su obra "El contrato social de 1762", establece que la soberanía se origina en el pueblo.

Para el tema en cuestión, el cual aborda la coyuntura de la globalización y por lo tanto las relaciones internacionales e intergubernamentales, la soberanía va más allá de sus fronteras territoriales. Así pues, cuestiones como los recursos transfronterizos, el comercio internacional, los derechos humanos y el medio ambiente, actualmente sobrepasa a los territorios para convertirse en un interés de todos los Estados en su conjunto, que a su vez hacen parte de organismos internacionales que tienen una competencia fraccionada, pues sus capacidades no son totalizantes, sino que se dividen en diferentes entes intergubernamentales con objetivos $\mathrm{y}$ alcances peculiarmente delineados. En el contexto actual de la globalización, se puede definir a la soberanía como "aquel poder que tiene el Estado para autodeterminarse en sus asuntos internos y para defender su independencia e igualdad ante otros Estados igualmente soberanos; es decir que el poder que tiene el Estado se entiende de dos formas: el interno y el internacional" (Becerra, Povedano y Téllez, 2016, p. 63).

La Educación Ambiental puede definirse como un proceso de formación educativa integral "que intenta mejorar las relaciones de los seres humanos con el medio a través del conocimiento y la sensibilización" (Calvo y Corraliza 1994, p. 18). De este modo, la EA “es un proceso permanente en el cual los individuos y las comunidades adquieren conciencia de su ambiente, aprenden los conocimientos y valores para actuar, individual y colectivamente, en la resolución de los problemas ambientales presentes y futuros" (Martínez, 2010, p. 100). La EA “debe abrir un proceso de construcción y apropiación de conceptos que generan sentidos divergentes sobre la sustentabilidad, implicando la necesidad de orientar un desarrollo en bases ecológicas, de equidad social, diversidad cultural y democracia participativa" (Leff, 1997, p. 21). Esto sugiere una Educación Ambiental que genere conciencia y capacidades propias para que las poblaciones puedan apropiarse de su ambiente como una riqueza económica (Leff, 1997, p. 22).

De acuerdo con García, existen tres tendencias clásicas de la enseñanza de la 
EA: Educación sobre el medio, en el medio y para el medio. La primera toma al medio natural como objeto de estudio dándole exclusividad a la ecología. La segunda toma al medio natural como recurso y la tercera, plantea al medio como algo a lo que hay que proteger y cuidar (García, 2004).

De acuerdo a lo anterior, la educación en, sobre y para el medio son concepciones camufladas en el antropocentrismo porque denotan la superioridad del ser humano sobre la naturaleza al concebirla como un recurso para su supervivencia o como algo digno de brindar protección. Si bien, el enfoque antropocéntrico ubica al ser humano distinto, superior, autónomo e independiente de las otras especies con el suficiente poder para conquistar y dominar al resto del planeta sin límite alguno. Este paradigma ignora en su totalidad la interdependencia y la complementariedad concebidas en el enfoque biocéntrico, donde involucra al ser humano dependiente de otras formas de vida en el planeta para poder subsistir. Supone una concepción sistémica donde los seres humanos hacemos parte de ese todo que es la biósfera. En este sentido, aprender a vernos como seres interdependientes del mundo biótico y abiótico, sugiere asumir una postura crítica desvinculada de los preceptos paternalistas de ver a la naturaleza digna de un cuidado el cual se ha establecido desde la década del $70 \mathrm{del}$ siglo XX, para ello se hace necesario acudir a la construcción social del conocimiento que, desde las aulas de clase les ayude a las futuras generaciones a concebir el mundo físico y el mundo social como uno solo.
Desde esta perspectiva, el Sur global requiere con urgencia una $\mathrm{EA}$ con una posición crítica y política frente a las formas de concentración de capital natural, lo cual busca mercantilizar absolutamente todo, incluyendo los bienes comunes como los ríos y las semillas, así como también, los bienes de acceso público como la atmósfera y el oxígeno, ejerciendo mecanismos de poder y dominación mediante un discurso legislativo que lo que busca es perpetuar la dominación de unos sobre otros; en este caso, mediante la concentración la naturaleza para el Norte y la estandarización de las externalidades negativas para el Sur. Una EA ambiental adecuada para el Sur implicaría la formación de educandos con un conocimiento integral de la realidad donde involucre el análisis de los elementos naturales insertados dentro de los procesos políticos, económicos, históricos y culturales que son propios de una sociedad. Desde esta perspectiva, se requiere una EA con un componente educativo que involucre una fuerte "concepción humanista y liberadora, exigiendo una postura reflexiva del educando" (Freire, 1970, p. 27).

\section{Las Cumbres internacionales de Estocolmo y Rio y la legislación colombiana con relación a la Educación Ambiental}

La Conferencia de las Naciones Unidas sobre el Medio Humano también llamada Cumbre de Estocolmo, fue celebrada en Suecia entre el 5 y el 16 de junio de 1972; marcando el inicio de las conferencias impartidas por la ONU en materia ambiental. Esta Cumbre contó con la 
representación de 113 países, 400 organizaciones no gubernamentales y 19 organismos intergubernamentales. El producto de esta reunión fue una Declaración que contiene 26 principios no vinculantes sobre el medio ambiente y el desarrollo, así como también, un plan de acción con 109 recomendaciones, estipuladas en la Resolución 2994 del 15 de diciembre de 1972. (Cabrera, 2003).

De los 26 principios de la Declaración de Estocolmo sobre Medio Ambiente Humano, el número 19 está correlacionado con la Educación Ambiental:

Es indispensable una labor de educación en cuestiones ambientales, dirigida tanto a las generaciones jóvenes como a los adultos y que preste la debida atención al sector de población menos privilegiado, para ensanchar las bases de una opinión pública bien informada, y de una conducta de los individuos, de las empresas y de las colectividades inspirada en el sentido de su responsabilidad en cuanto a la protección y mejoramiento del medio ambiente en toda su dimensión humana. Es también esencial que los medios de comunicación de masas eviten contribuir al deterioro del medio ambiente humano y difundan, por el contrario, información de carácter educativo sobre la necesidad de protegerlo y mejorarlo, a fin de que el hombre pueda desarrollarse en todos los aspectos. (Principio 19, Naciones Unidas, 1972, p. 3).

Como resultado institucional de esta Cumbre, nace el Programa de las Naciones
Unidas sobre Ambiente (PNUMA); órgano subsidiario de la Asamblea general, que a pesar de carecer de recursos humanos y financieros y de estar lejos de los centros operativos de las Naciones Unidas, "el PNUMA ha sido capaz de desempeñar una labor importante porque ha servido de impulso decisivo para la celebración de los acuerdos ambientales multilaterales universales" (Cardesa, 2011).

Veinte años después de la Cumbre de Estocolmo, se celebra del 2 al 14 de junio de 1992 en Río de Janeiro (Brasil), la Conferencia de Naciones Unidas sobre Medio Ambiente y Desarrollo, conocida también como Cumbre de Río o Cumbre de la tierra. El resultado de esta reunión estipulada por 178 gobiernos, fue, por un lado, la creación de la Comisión de Desarrollo Sostenible y por otro, la elaboración de varios documentos jurídicos como la Agenda 21, la Convención sobre la Diversidad Biológica, el Convenio Marco de Cambio Climático y la Declaración de Río, la cual está constituida por veintisiete principios donde el número diez es el que está directamente involucrado con la Educación ambiental:

El mejor modo de tratar las cuestiones ambientales es con la participación de todos los ciudadanos interesados, en el nivel que corresponda. En el plano nacional, toda persona deber tener acceso adecuado a la información sobre el medio ambiente de que dispongan las autoridades públicas, incluida la información sobre los materiales y las actividades que 
encierran peligro en sus comunidades, así como la oportunidad de participar en los procesos de adopción de decisiones. Los Estados deberán facilitar y fomentar la sensibilización y la participación de la población poniendo la información a disposición de todos. Debe proporcionarse acceso efectivo a los procedimientos judiciales y administrativos, entre estos el resarcimiento de daños y los recursos pertinentes. (Principio 10, Naciones Unidas, 1992, p. 3).

Como resultado de la Cumbre de Estocolmo, en el contexto colombiano, emerge la Ley 23 de 1973, la cual establece que "el medio ambiente está constituido por la atmósfera y los recursos naturales renovables" (Congreso de Colombia Ley 23 de 1973, p. 1). Así mismo, concede facultades extraordinarias al Presidente de la República para expedir el Código de Recursos Naturales y protección al medio ambiente; conllevando a que al siguiente año de su reglamentación y bajo el mandato del militante liberal Alfonso López Michelsen (1974-1978), se expidiera el Decreto-Ley 2811 de 1974, cuyo texto también acoge los presupuestos conservacionistas y preservacionistas de la Cumbre de Estocolmo. En este sentido, con el Código Nacional de Recursos Naturales Renovables y de Protección al Medio Ambiente se "armonizó la legislación dispersa existente en el momento y dispuso la gestión ambiental en cabeza del ejecutivo. Por lo tanto, es el escenario donde inicia el Derecho ambiental en Colombia" (Alvarado, 2015, p. 141).
Si bien, luego de su expedición en 1974, el Decreto-Ley 2811 tardaría cerca de tres años en lograr su implementación, el cual no hubiese sido posible gracias a un grupo de ambientalistas que propiciaron en el país respuestas positivas a la Conferencia de Estocolmo. En este sentido, actores sociales como Julio Carrizosa, quien fue director del desaparecido Inderena y Miguel Urrutia, jefe de planeación de la época, entre otros, "avanzaron en la gestión ambiental durante el gobierno de López Michelsen, producto de la tradición del liberalismo filosófico y de su hijo, el pragmatismo" (Carrizosa, 2008, p. 27); en un momento de la historia colombiana donde se da inicio a una nueva época, luego de la culminación en 1974 del acuerdo bipartidista de alternancia del poder denominado Frente Nacional.

Con un total de 340 artículos, el Código Nacional de los Recursos Naturales Renovables establece al ambiente como un patrimonio común de la humanidad y tiene por objeto alcanzar la preservación, conservación y restauración del medio ambiente y la utilización racional de los recursos naturales. Así mismo, en el artículo 14 de este Decreto se inserta el componente educativo a saber:

Dentro de las facultades que constitucionalmente le competen, el gobierno al reglamentar la educación primaria, secundaria y universitaria, procurará: a) Incluir cursos sobre ecología, preservación ambiental y recursos naturales renovables; b) Fomentar el desarrollo de estudios interdisciplinarios; c) Promover la realización de jornadas ambientales 
con participación de la comunidad, y de campañas de educación popular, en los medios urbanos y rurales para lograr la comprensión de los problemas del ambiente, dentro del ámbito en el cual se presentan (Artículo 10, Presidencia de la República de Colombia, 1974, p. 5).

Así las cosas, una vez de haberse expedido el código Nacional de los Recursos Naturales Renovables, diecisiete años después, el país fortaleció su normatividad ambiental a través de la Constitución de 1991; Carta política que le dio un reconocimiento significativo al medio ambiente $\mathrm{y}$, de manera similar a las reformas institucionales de los años setenta que fueron respuesta a la Conferencia de Estocolmo, el 22 de diciembre de 1993 se expide en Colombia la Ley 99, como una réplica a los compromisos adquiridos en la Conferencia de las Naciones Unidas, efectuada en Río de Janeiro en el año 1992.

Si bien, la Ley 99 de 1993 nace durante el mandato del presidente liberal César Augusto Gaviria Trujillo (1990-1994), quien tuvo una firme decisión de sacarla adelante con el apoyo de una gran coalición de ambientalistas, entre ellos, funcionarios del gobierno y ONGs de la época, a pesar de la negativa de ciertos sectores del petróleo, de la construcción y de la ANDI quienes fueron opositores al proyecto de Ley. Sin embargo, Cesar Gaviria "participó activamente para llevar a cabo la instauración de la Ley 99, como una manera de dar respuesta a los acuerdos firmados por más de ciento veinte jefes de
Estado en la denominada Cumbre de la Tierra de 1992" (Rodríguez, 2009, p. 22).

En este sentido, la Ley 99, reúne en su totalidad elementos constitutivos de la Declaración de Río, apuntando al desarrollo económico y social de Colombia, la protección y conservación del ambiente, el desarrollo sostenible, la creación del Ministerio del Ambiente y del Sistema Nacional Ambiental (SINA), e involucra la implementación de la EA por parte de los Ministerios del Medio Ambiente y de Educación Nacional:

Corresponde al Ministerio del Medio Ambiente: Adoptar, conjuntamente con el Ministerio de Educación Nacional, a partir de enero de 1995, los planes y programas docentes y el pénsum que en los distintos niveles de la educación nacional se adelantarán en relación con el medio ambiente y los recursos naturales renovables, promover con dicho Ministerio programas de divulgación y educación no formal y reglamentar la prestación del servicio ambiental. (Congreso de Colombia. Artículo 5, numeral 9 de la Ley 99 de 1993).

Así mismo, como resultado de la función asignada a los Ministerios del Medio Ambiente y de Educación Nacional, estipuladas en la Ley 99 de 1993 con respecto a la EA, nace el 5 de agosto de 1994, el Decreto 1743 el cual institucionaliza en todos los establecimientos de educación formal del país, tanto oficiales como privados, los proyectos ambientales, escolares (PRAES), con la finalidad de "formar a los 
educandos en la protección, preservación y aprovechamiento de los recursos naturales y el mejoramiento de las condiciones humanas y del ambiente" (Diario Oficial no 41.476, Decreto 1743 de 1994, Ministerio de Educación Nacional de Colombia).

De este modo, vemos cómo las Cumbres de Estocolmo y de Rio producen discursos desde del Norte para ser reproducidos en el Sur global, en este caso en el contexto colombiano a través de su jurisprudencia, en materia de EA. Luego de explorar el marco teórico en cuestión, se pasará a realizar el análisis discursivo de estas producciones $\mathrm{y}$ reproducciones legislativas.

\section{Hacia un análisis del discurso: Producción enunciativa preservacionista y conservacionista de la Cumbre de Estocolmo y su reproducción discursiva en la legislación colombiana en el Decreto 2811 de 1974}

Retomando nuevamente los presupuestos de Mendizábal, quien aborda el enfoque del discurso en diversos paquetes denominados textos y donde las Cumbres en cuestión son textos sociales, el autor plantea que "El texto está ubicado dentro de un contexto que le explica y le determina”. (Mendizábal, 1999, p. 117). Así pues, ambas Cumbres hacen parte de contextos históricos y políticos que son propios de la época y que por lo tanto ayudan a crear y recrear dentro de sus discursivas, elementos propios del contexto. Por ejemplo, la Cumbre de Estocolmo, la cual estuvo fuertemente influenciada por el reporte "Los límites del
Crecimiento", informe del Club de Roma, asociación privada compuesta por empresarios, científicos y políticos, pone de relieve las limitaciones de los recursos naturales y la importancia de conservarlos porque "los procesos productivos descansan en la naturaleza. Ésta que siempre estuvo por fuera de la temática del desarrollo, repentinamente toma un papel central en la discusión" (Gudynas, 1999, p. 110). Desde esta perspectiva, la Cumbre de Estocolmo toma un énfasis discursivo conservacionista y preservacionista de los recursos naturales por las predicciones del Club Roma, cuyos estudios indicaban que en 30 años se produciría una grave crisis en las producciones industriales $y$ agrícolas (Zapiain, 2003, p. 1).

Todo apunta a la necesidad de crear narrativas que ayuden a conservar y preservar la naturaleza para que el Norte global pueda continuar con la estandarización de sus procesos productivos y de sus exorbitantes hábitos de consumo, mientras que el Sur global por medio de leyes, mandatos o marcos legales reproduce dentro de su territorio la perpetuación enunciativa impuesta desde del Norte global. Lo anterior hace referencia al "orden del discurso" planteado por Foucault el cual pone en acción la producción de saberes y prácticas que, en este caso, van al servicio de la hegemonía imperial. Como ejemplificación de lo anterior, Colombia, país perteneciente al Sur global, establece en el Decreto 2811 de 1974 denominado Código Nacional de los Recursos Naturales Renovables, discursivas que conllevan al cuidado, preservación, conservación y uso racional de los 
recursos naturales, involucrando a su población e incluyendo a las futuras generaciones a insertarse en lógicas preservacionistas encaminadas al servicio del capital.

Así mismo, la Cumbre de Estocolmo se enmarca dentro del contexto de la guerra fría, donde los países del bloque occidental capitalista buscan en la idea del desarrollo económico una estrategia para fortalecer su posición frente a los países del bloque oriental. Esta táctica discursiva llevada a cabo por los países del Norte, busca llevar bienestar a todos los rincones del mundo erradicando la pobreza, la cual es vista a partir de los años cuarenta y cincuenta como la causante de todos los males, entre ellos, de la degradación ambiental. Desde esta perspectiva, La Cumbre de Estocolmo es la antesala para pensar la naturaleza desde el discurso del desarrollo sostenible, el cual se consolida 15 años después con el Informe Brundtland, documento emanado en 1987; también denominado "Nuestro Futuro Común" el cual busca yuxtaponer dos construcciones abstractas: desarrollo y sostenibilidad.

\section{Hacia un análisis del discurso: Producción enunciativa del desarrollo en la Cumbre de Río y su reproducción en la legislación colombiana con la Ley 99 de 1993 y el Decreto 1743 de 1994}

De acuerdo con el contexto que le explica y le determina, la Cumbre de Río realizada 20 años después de la Cumbre de Estocolmo, se enmarca en una época neoliberal. Con la desaparición del mundo socialista soviético bajo el símbolo de la caída del muro de Berlín en 1989, se tiene la esperanza de construir un 'mundo nuevo' abriendo 'los cauces en el mercado mediante la liberalización y desregulación de la economía, para que los desequilibrios se corrigieran y se iniciara una nueva era de crecimiento sostenido" (Guillén, 2007, p. 490).

En este sentido, la Cumbre de las Naciones Unidas para el Medio Ambiente y el Desarrollo, involucra desde su discursividad, la importancia del crecimiento económico a través de un sistema económico internacional que conlleve al desarrollo sostenible, concepto que se comienza a concebir desde el informe del Club Roma, se gesta en la conferencia de las Naciones Unidas, nace con el Informe Brundtland y se consolida en la Cumbre de Río, enmarcada desde una perspectiva dominante globalocéntrica, como una forma de bioimperalismo que busca en realidad reafirmar los intereses del Norte, desatendiendo cuestiones profundas de la degradación ambiental (Escobar, 2007).

Desde esta perspectiva, con Ley 99 de 1993 y el Decreto 1743 de 1994, se perfila y se reproduce el discurso antropocéntrico con relación al desarrollo económico y social de Colombia, dogmatizando el concepto de desarrollo sostenible a través de la normatividad colombiana, introduciéndolo en las conciencias de los estudiantes a través de la implementación de los PRAES en las instituciones educativas públicas y privadas del todo el país. Una forma de biopoder institucionalizada a través del aparato educativo para el control total de los cuerpos y la reproducción discursiva de que el desarrollo será la panacea a todos los males del país. De acuerdo con Shiva, (1989), "el concepto de desarrollo no es 
más que un discurso totalizante y hegemónico que perpetúa las desigualdades económicas entre Norte y Sur" (Citado en Arboleda Márquez, 2010, p. 19).

De acuerdo a todo lo anterior, se va estructurando todo un discurso revestido de doble moral donde los países del Norte fomentan condiciones que le son favorables para sus intereses de dominio como una nueva forma de colonialidad que ya no "opera por medio de la conquista, sino a través de la imposición de normas como mercados libres, nociones culturales de consumo, democracias al estilo de Estados Unidos y, tratados ambientales globales" (Escobar, 2012, p. 395).

Como se puede apreciar, en la discursividad de los dos tratados internacionales con relación a la EA, éstos no han evolucionado considerablemente, preservándose la retórica conservacionista y pasiva de que la naturaleza es una entidad a la cual se debe cuidar y preservar para que esté al servicio del capital. Así mismo, el desarrollo sostenible se ha constituido como un paradigma dogmático para solucionar los problemas de pobreza, de hambre y de inequidad que hay en el mundo. El análisis histórico, geopolítico y discursivo permite evaluar que toda la discursividad entretejida con relación al desarrollo no ha permitido ni distribuir la riqueza ni mejorar las condiciones de vida de muchas personas en el mundo. El bienestar económico y ecológico se está concentrando cada vez más en el Norte global; mientras que en el Sur se están acumulando las externalidades negativas.

\section{Conclusiones}

Con la nueva configuración del mundo ya no se puede hablar de una soberanía totalizante por parte de los Estados, sino que éstos comparten ciertos asuntos que van más allá de sus fronteras territoriales. Cuestiones como los derechos humanos o el medio ambiente, hacen parte de la nueva gobernanza internacional la cual crea y recrea discursos que son producidos en el Norte y reproducidos en el Sur. En este sentido, el lugar de enunciación, el contexto histórico-social y los acuerdos establecidos desde el Norte global, son acogidos por los diversos Estados a través de la creación de Leyes y Decretos que lo que buscan es disciplinar los cuerpos y moldear conductas.

Como ejemplo de lo anterior, en Colombia, diversos actores sociales a través de la legislación colombiana, han reproducido al interior de sus leyes, discursos que fueron creados desde el Norte, en este caso, a través de las Cumbres de Estocolmo y de Río para ser reproducidas en el aparato educativo del país por medio de la implementación del Decreto 2811 de 1974, de la Ley 99 de 1993 y, en especial del Decreto 1743 de 1994, el cual materializa dicha discursiva a través de la implementación de los PRAES en las instituciones educativas públicas y privadas de Colombia. En estas narrativas jurisprudenciales, se aborda la visión antropocéntrica de que todos los colombianos tenemos la responsabilidad de cuidar y preservar la naturaleza y buscar el desarrollo sostenible a través de la EA, dejando de lado la construcción crítica y política de una comunidad con relación a su ambiente, su naturaleza, su historia y su 
cultura, ignorando hábitos de consumo y producción, así como también, de concentración y acumulación de capital. En este sentido, el Sur global requiere otra clase de EA y no la recreada por el Norte a través de sus Cumbres o Conferencias internacionales, las cuales representan unos intereses intrínsecos: ¿Preservar y cuidar la naturaleza para qué? ¿Buscar el desarrollo sostenible para quién? ¿Quiénes han sido los verdaderos beneficiados frente a estas prácticas discursivas? ¿De qué ha servido el desarrollo sostenible para solucionar los problemas de hambre e inequidad existentes y perdurables en el Sur global?

\section{Referencias bibliográficas}

Aldo Isuani, E. (2011). Tres enfoques sobre el concepto de Estado (Trabajo de Maestría en Administración Pública). Universidad de Buenos Aires. http://www.aldoisuani.com/wpcontent/uploads/2011/01/TresEnfoques-sobre-el-concepto-deEstado11.pdf

Alvarado, J, C. (2015). La protección de la naturaleza y el ambiente, un nuevo objetivo del ordenamiento jurídico. Análisis comparativo entre Colombia y Francia. Verba Luris, (33), 137-148. https://doi.org/10.18041/01213474/verbaiuris.33.31

Arboleda Márquez, M. (2010). El postestructuralismo como punto de intersección entre medio ambiente y sociedad. Civilizar, Ciencias Sociales y Humanas, 10(19), 1522. https://doi.org/10.22518/1657895 3.47

Becerra Ramírez, M., Povedano Amezola, A., y Téllez Carvajal, E. (2016). La soberanía en la era de la globalización. En Soberanía y juridificación en las relaciones internacionales.

https://archivos.juridicas.unam.mx /www/bjv/libros/6/2790/5.pdf

Cabrera Medaglia, J. (2003). El impacto de las declaraciones de Río y Estocolmo sobre la legislación y las políticas ambientales en América Latina. Revista de Ciencias Jurídicas, (100), p. 301-331.

https://revistas.ucr.ac.cr/index.php /juridicas/article/view/13406/1266 8

Calduch, R. (1991). El Estado, el Pueblo y la Nación. En Relaciones Internacionales. Madrid. https://www.ucm.es/data/cont/me dia/www/pag-55159/lib1cap6.pdf

Calvo, S., y Corraliza, J., A. (1994). Introducción a la Educación Ambiental. En Educación ambiental: conceptos y propuestas (pp. 9-21). Madrid: Editorial CCS.

Campos Fernández, E. (2010). Reseña Historia de la sexualidad 1: La voluntad del saber de Michel Foucault. Sapiens. Revista Universitaria de Investigación, 11(1), 231-233. http://www.redalyc.org/pdf/410/4 1021794014.pdf

Cardesa Salzmann, A. (2011). La configuración de los acuerdos ambientales universales como regímenes autónomos de control y 
la institucionalización de los acuerdos ambientales universales. En El control internacional de aplicación de los acuerdos ambientales universales (pp. 1393). España: Marcial Pons.

Carrizosa, J. (2008). Prólogo. Desarrollo y ambiente. En Gobernabilidad, instituciones y medio ambiente en Colombia, ed. Manuel Rodríguez Becerra, 1-64. Bogotá: FNA.

Congreso de Colombia. (19 de diciembre de 1973). Ley 23: Por el cual se conceden facultades extraordinarias al Presidente de la República para expedir el Código de Recursos Naturales y de Protección al Medio Ambiente y se dictan otras disposiciones. https://www.funcionpublica.gov.c o/eva/gestornormativo/norma.php ? $\mathrm{i}=9018$

Congreso de Colombia. (22 de diciembre de 1993). Ley 100: Por la cual se crea el Ministerio del Medio Ambiente, se reordena el Sector Público encargado de la gestión y conservación del medio ambiente y los recursos naturales renovables, se organiza el Sistema Nacional Ambiental, SINA y se dictan otras disposiciones.

http://www.secretariasenado.gov.c o/senado/basedoc/ley_0099_1993. html

Escobar, A. (2007). La invención del Tercer Mundo Construcción y deconstrucción del desarrollo. Fundación Editorial el perro y la rana. Caracas, Venezuela.

Escobar, A. (2012). Más allá del tercer mundo: Globalidad imperial, colonialidad global, y movimientos sociales antiglobalización. En Antropología y desarrollo. Discursos, prácticas y actores, Editorial Los Libros de la Catarata (pp. 386-412). Madrid, España.

Freire, P. (1970). La educación como una dimensión de la acción cultural. En Sobre la acción cultural (pp. 1950). Instituto de Capacitación e Investigación en Reforma Agraria: Proyecto del Gobierno de Chile.

García, E. (2004). Educación ambiental, constructivismo y complejidad: una propuesta integradora. España: Editorial Díada.

Gudynas, E. (1999). Concepciones de la Naturaleza y Desarrollo en América Latina. Persona y Sociedad, 13(1), 101-125.

Guillén, A. (2007). La teoría latinoamericana del desarrollo. Reflexiones para una estrategia alternativa frente al neoliberalismo. En G. Vidal, y A. R. Guillén R. (Comps.), Repensar la teoría del desarrollo en un contexto de globalización. Homenaje a Celso Furtado.

Leff, E. (1997). Educación Ambiental y Desarrollo Sustentable. Revista formación ambiental, 9-10(20-24).

Martínez Castillo, R. (2010). La importancia de la educación ambiental ante la problemática actual. Revista Electrónica Educare, $\quad$ XIV(1), 97-111. http://www.redalyc.org/pdf/1941/ 194114419010.pdf. 
Mendizábal, I. (1999). Del análisis del contenido al análisis del discurso: Aspectos metodológicos en relación a la etnometodología. En Análisis del discurso social y político. Quito, Ecuador: Ediciones ABYA-YALA.

Ministerio de Educación Nacional. (03 de agosto de 1994). Decreto 1743: Por el cual se instituye el Proyecto de Educación Ambiental para todos los niveles de educación formal, se fijan criterios para la promoción de la educación ambiental no formal e informal $y$ se establecen los mecanismos de coordinación entre el Ministerio de Educación nacional y el Ministerio del Medio Ambiente.

https://www.minambiente.gov.co/i mages/BosquesBiodiversidadySer viciosEcosistemicos/pdf/Normativ a/Decretos/dec_1743_030894.pdf

Naciones Unidas. (1972). Declaración de Estocolmo sobre el medio ambiente humano del 16 de junio de 1972. http://www.ordenjuridico.gob.mx/ TratInt/Derechos\%20Humanos/IN ST\%2005.pdf

Naciones Unidas. (1992). Declaración de Rio sobre el medio ambiente y el desarrollo del 14 de junio de 1992. https://www.un.org/spanish/esa/su stdev/agenda21/riodeclaration.htm

Presidencia de la República. (18 de diciembre de 1974). Decreto 2811: Por el cual se dicta el Código Nacional de Recursos Naturales Renovables y de Protección al Medio Ambiente. http://www.secretariasenado.gov.c o/senado/basedoc/decreto_2811_1 974.html

Revel, J. (2008). Diccionario Foucault. Buenos Aires, Argentina: Ediciones Nueva Visión.

Rodríguez, M. (2009). ¿Hacer más verde al Estado colombiano? Revista de estudios sociales, (32), 18-33. https://journals.openedition.org/re vestudsoc/16205?lang=fr

Shiva, V. (1989). Staying Alive: Women Ecology and Development. London. Zed Books.

Vásquez Rocca, L. (2013). La noción de biopoder en Foucault y su relación con las antropotécnicas en la obra del último Sloterdijk. Eikasia, Revista de filosofía, (53), 61-74. http://www.revistadefilosofia.org/ 53-04.pdf

Zapiain Aizpuru, M. (2003). Los límites del crecimiento: informe al Club de Roma sobre el predicamento de la Humanidad. Reseña. http://habitat.aq.upm.es/gi/mve/da ee/tmzapiain.pdf 